Article

\title{
Optimization and a Kinetic Study on the Acidic Hydrolysis of Dialkyl $\alpha$-Hydroxybenzylphosphonates
}

\author{
Nikoletta Harsági ${ }^{1}$, Zita Rádai ${ }^{1}$, Áron Szigetvári ${ }^{2}$, János Kóti ${ }^{2}$ and György Keglevich ${ }^{1, *(D)}$ \\ 1 Department of Organic Chemistry and Technology, Budapest University of Technology and Economics, \\ 1521 Budapest, Hungary; harsagi.nikoletta@mail.bme.hu (N.H.); radai.zita@mail.bme.hu (Z.R.) \\ 2 Gedeon Richter Plc., 1475 Budapest, Hungary; szigetvaria@richter.hu (Á.S.); j.koti@richter.hu (J.K.) \\ * Correspondence: gkeglevich@mail.bme.hu; Tel.: +36-1-463-1111/5883
}

Academic Editors: Milos Sedlak

Received: 14 July 2020; Accepted: 17 August 2020; Published: 20 August 2020

\begin{abstract}
The two-step acidic hydrolysis of $\alpha$-hydroxybenzylphosphonates and a few related derivatives was monitored in order to determine the kinetics and to map the reactivity of the differently substituted phosphonates in hydrolysis. Electron-withdrawing substituents increased the rate, while electron-releasing ones slowed down the reaction. Both hydrolysis steps were characterized by pseudo-first-order rate constants. The fission of the second P-O-C bond was found to be the rate-determining step.
\end{abstract}

Keywords: dialkyl $\alpha$-hydroxyphosphonates; hydrolysis; phosphonic ester-acid intermediate; phosphonic acid; rate constants; mechanism

\section{Introduction}

The hydrolysis of P-esters (e.g., phosphinates and phosphonates) resulting in the formation of the corresponding acids (phosphinic acids and phosphonic acids, respectively) is an important chemical transformation, and hence it is applied widely in syntheses. Most often, the hydrolyses were performed under acidic conditions [1-4], but the application of $\mathrm{NaOH}$ or $\mathrm{KOH}$ is also common [5-8]. An additional possibility is the fission of the P-O-C unit by the effect of $\mathrm{Me}_{3} \mathrm{SiBr}$ [9-11]. Usually, the acid- or base-catalyzed hydrolyses were carried out routinely, under "excessive" (unoptimized) conditions applying the acid or base catalysts in a larger quantity than required, and allowing longer reaction times. We undertook to explore the optimum conditions for the $\mathrm{HCl}$-catalyzed hydrolysis of phosphinic and phosphonic esters. In the first round, the acid-catalyzed hydrolysis of cyclic phosphinates, such as 1-alkoxy-3-phospholene oxides, 1-alkoxyphospholane oxides, and an 1-alkoxy-1,2,3,4,5,6-hexahydrophosphinine oxide was investigated, optimized, and characterized by rate constants [12]. Then, the hydrolysis of a series of dialkyl arylphosphonates was studied. In this case, two-step conversions were monitored and quantified by k values [13]. $\alpha$-Hydroxybenzylyphosphonates, obtained in the Pudovik reaction of substituted benzaldehydes and dialkyl phosphites, form a representative class of phosphonic acid derivatives [14]. $\alpha$-Hydroxyphosphonates are versatile intermediates that may be transformed to $\alpha$-aminophosphonates [15], can be phosphorylated [16], and may be rearranged to the corresponding phosphates [17]. The catalytic hydrogenation of $\alpha$-dibenzyl hydroxyphosphonates afforded the respective $\alpha$-hydroxyphosphonic acids [18]. Moreover, they may be of cytotoxic activity [18]. In this article, we describe our results on the $\mathrm{HCl}$-promoted hydrolysis of $\alpha$-hydroxyphosphonates and a few related analogues.

\section{Results and Discussion}

Substituted $\alpha$-hydroxybenzylphosphonates $(\mathbf{1} \mathbf{a}-\mathbf{j})$ prepared as described earlier [18] were subjected to acidic hydrolysis. The application of three equivalents $(0.5 \mathrm{~mL})$ of concentrated hydrochloric acid in 
$1 \mathrm{~mL}$ of water for ca. $2 \mathrm{mmol}$ of the phosphonate (1) at reflux resulted in complete hydrolysis within 2.5-9.5 h depending on the substituents. The reactions followed a two-step protocol and took place via the corresponding ester-acid intermediate $\mathbf{2}$, and were monitored by ${ }^{31} \mathrm{P}$ NMR spectroscopy (Scheme 1 ). Experimental data together with the calculated pseudo-first-order $\mathrm{k}_{1}$ and $\mathrm{k}_{2}$ rate constants are listed in Table 1, while the concentration-time diagrams exhibiting the relative proportions of components $\mathbf{1}, \mathbf{2}$, and 3 are shown in Figures 1 and 2 and Figures S1-S8 in the Supplementary Materials.<smiles>[Y]c1ccc(C(O)P([R])([R])=O)cc1</smiles><smiles>[Y]c1ccc(C(O)P([R])(=O)O)cc1</smiles><smiles>[Y]c1ccc(C(O)P(=O)(O)O)cc1</smiles>

Scheme 1. Two-step acidic hydrolysis of substituted $\alpha$-hydroxybenzylphosphonates. For the Y substituents see Table 1.

Table 1. Experimental and kinetic data on the two-step hydrolysis of $\alpha$-hydroxybenzylphosphonates $1 \mathbf{a}-\mathbf{j}$.

\begin{tabular}{cccccccc}
\hline Entry & $\mathbf{Y}$ & $\mathbf{R}$ & $\boldsymbol{t}_{\text {max }}(\mathbf{m i n})$ & $\mathbf{t}_{\mathbf{r}}(\mathbf{h})$ & $\mathbf{k}_{\mathbf{1}}\left(\mathbf{h}^{\mathbf{- 1}}\right)$ & $\mathbf{k}_{\mathbf{2}} \mathbf{( \mathbf { h } ^ { - \mathbf { 1 } } )}$ & $\mathbf{R}^{\mathbf{2}}$ \\
\hline 1 & $\mathrm{H} \mathrm{(a)}$ & $\mathrm{Me}$ & 44 & 6.5 & 2.64 & 0.60 & 0.994 \\
2 & $\mathrm{NO}_{2}(\mathbf{b})$ & $\mathrm{Me}$ & 22 & 2.5 & 5.18 & 1.24 & 0.989 \\
3 & $\mathrm{Cl}(\mathbf{c})$ & $\mathrm{Me}$ & 34 & 5.5 & 3.36 & 0.79 & 0.987 \\
4 & $\mathrm{~F}(\mathbf{d})$ & $\mathrm{Me}$ & 32 & 6.0 & 3.93 & 0.67 & 0.965 \\
5 & $\mathrm{CF}_{3}(\mathbf{e})$ & $\mathrm{Me}$ & 51 & 5.5 & 2.03 & 0.61 & 0.988 \\
6 & $\mathrm{Me}(\mathbf{f})$ & $\mathrm{Me}$ & 76 & 8 & 1.64 & 0.31 & 0.962 \\
7 & $\mathrm{H} \mathrm{(g)}$ & $\mathrm{Et}$ & 90 & 9.5 & 1.03 & 0.35 & 0.986 \\
8 & $\mathrm{NO}(\mathbf{h})$ & $\mathrm{Et}$ & 75 & 5.5 & 1.40 & 0.61 & 0.992 \\
9 & $\mathrm{Cl}(\mathbf{i})$ & $\mathrm{Et}$ & 60 & 8.0 & 1.08 & 0.42 & 0.992 \\
10 & $\mathrm{~F}(\mathbf{j})$ & $\mathrm{Et}$ & 80 & 9.0 & 1.35 & 0.31 & 0.970 \\
\hline
\end{tabular}

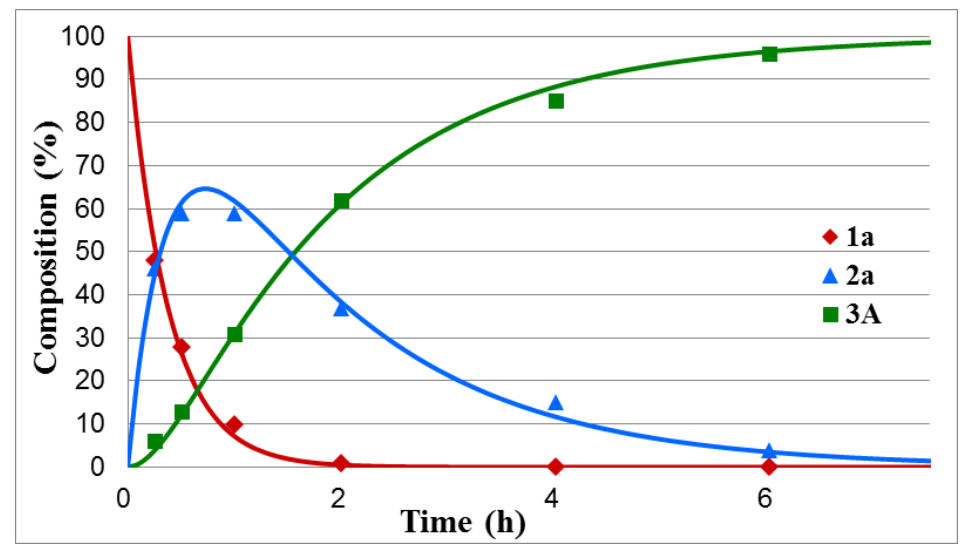

Figure 1. Concentration profile for the components during the hydrolysis of dimethyl $\alpha$ hydroxybenzylphosphonate (1a) under optimum conditions. The $R^{2}$ measure of goodness of fit is 0.994 . 


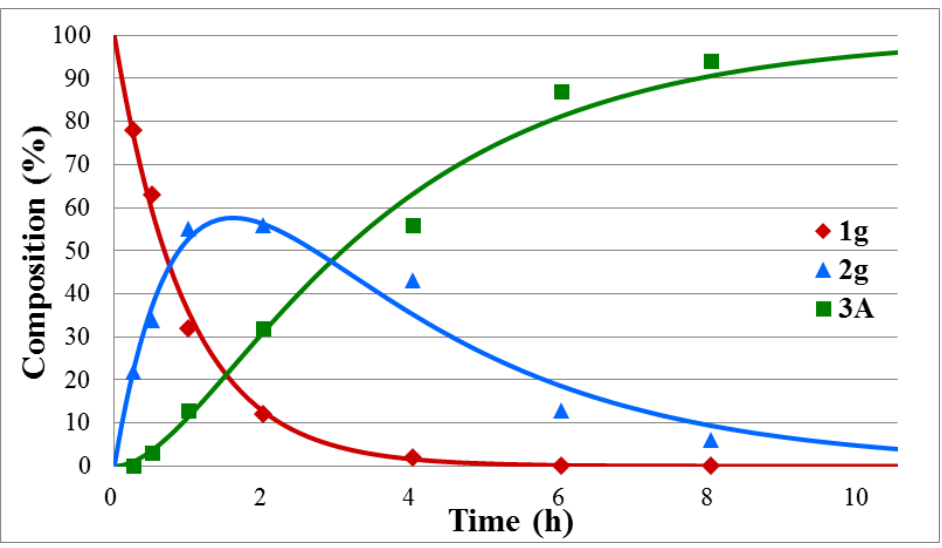

Figure 2. Concentration profile for the components during the hydrolysis of diethyl $\alpha$ hydroxybenzylphosphonate $(\mathbf{1 g})$ under optimum conditions. The $\mathrm{R}^{2}$ measure of goodness of fit is 0.986 .

One can see that the hydrolysis of the unsubstituted dimethyl $\alpha$-hydroxybenzylphosphonate was complete after $t_{r}=6.5 \mathrm{~h}$, and the maximum proportion of intermediate $2 \mathrm{a}$ could be observed at $t_{\max }=44 \mathrm{~min}$. In this case, $\mathrm{k}_{1}$ and $\mathrm{k}_{2}$ were found to be $2.64 \mathrm{~h}^{-1}$ and $0.60 \mathrm{~h}^{-1}$, respectively (Table 1/Entry 1). Electron-withdrawing substituents, such as 4- $\mathrm{NO}_{2}, 4-\mathrm{Cl}$ and 4-F in the phenyl ring facilitated the hydrolyses that were complete after $2.5 \mathrm{~h}, 5.5 \mathrm{~h}$, and $6 \mathrm{~h}$, respectively. The maximum concentration of intermediates $\mathbf{2 b}-\mathbf{d}$ appeared in the range of $22-34 \mathrm{~min}$. The $\mathrm{k}_{1}$ values fell in the range of 3.36-5.18 $\mathrm{h}^{-1}$, while the $\mathrm{k}_{2}$ constants were between 0.67 and $1.24 \mathrm{~h}^{-1}$ (Table 1/Entries 2-4). It seems that the $4-\mathrm{CF}_{3} \mathrm{Ph}$ substituent acted overall as the $4-\mathrm{ClPh}$ group, as marked by $\mathrm{t}_{\mathrm{r}}=5.5 \mathrm{~h}$. In this case $\left(\mathrm{Y}=\mathrm{CF}_{3}\right), \mathrm{k}_{1}$ was 2.03, while $\mathrm{k}_{2}$ was 0.61 (Table 1/Entry 5). In the above series, the hydrolysis of the 4-Me-substituted benzylphosphonate (1f) was the slowest, as a complete hydrolysis required 8 $\mathrm{h}$, and the rate constants were found to be $1.64\left(\mathrm{k}_{1}\right)$ and $0.31\left(\mathrm{k}_{2}\right)$ (Table 1/Entry 6). It is noteworthy that the fission of the second P-OMe unit is the rate-determining step. As can be seen from Table 1 , the $k_{1}$ values for the above cases are, in almost all cases, more than four times larger as compared to the $\mathrm{k}_{2}$ values.

Regarding the series of substituted diethyl $\alpha$-hydroxybenzylphosphonates $(\mathbf{1} \mathbf{g}-\mathbf{j})$, the hydrolysis of the unsubstituted model $(\mathbf{1 g})$ was significantly slower than that of the dimethyl analogue (1a) (compare the reaction times of $9.5 \mathrm{~h}$ (Table 1/Entry 7) and $6.5 \mathrm{~h}$ (Table 1/Entry 1 )). The corresponding $\mathrm{k}_{1}$ and $\mathrm{k}_{2}$ rate constants for the hydrolysis of diethyl ester $1 \mathrm{~g}$ were roughly the half the ones obtained for the methyl counterpart (1a) (compare rate constants 1.03/0.35 versus 2.64/0.60 (Table 1/Entry 7 versus Entry 1). Hydrolysis of the $\alpha$-hydroxyphosphonates with electron-withdrawing $4-\mathrm{NO}_{2}, 4-\mathrm{Cl}$, and 4-F substituents in the phenyl ring $(\mathbf{1 h}-\mathbf{j})$ required shorter reaction times of 5.5-9.0 $\mathrm{h}$ as compared with that $(9.5 \mathrm{~h})$ of the unsubstituted instance $(\mathbf{1 g})$ (Table 1/Entries $8-10)$. In these cases again, the $\mathrm{k}_{2}$ rate constants $(0.61,0.42$, and 0.31 , respectively) determined the overall reactivity.

To study the effect of substituents on the rate of the hydrolysis further, three additional model compounds, diethyl benzylphosphonate (4k), diethyl $\alpha$-phenylethylphosphonate (41), and diethyl $\beta$-phenylethylphosphonate (4m) were also subjected to hydrolysis, under the conditions applied for the $\alpha$-hydroxybenzylphosphonates (1a-j) above (Scheme 2, Table 2, Figure 3 and Figures S9 and S10 in Supplementary Materials). It was found that the hydrolysis of the benzylphosphonate (4k) took longer than that of the $\alpha$-hydroxy derivative $1 \mathbf{g}$ (15 h versus $9.5 \mathrm{~h}$, Table 2/Entry 1 and Table 1/Entry 7). The $\mathrm{k}_{1}$ constant was somewhat larger for the hydrolysis of species $\mathbf{4 k}$ than that for $\mathbf{1 g}$, but the decisive $\mathrm{k}_{2}$ value become lower, as demonstrated by $1.12 \mathrm{~h}^{-1}$ and $0.20 \mathrm{~h}^{-1}$ versus $1.03 \mathrm{~h}^{-1}$ and $0.35 \mathrm{~h}^{-1}$ data pairs, respectively. Placing a Me group instead of the $\mathrm{OH}$ function on the $\alpha \mathrm{C}$ atom, i.e., starting from $\alpha$-phenylethylphosphonate 41 , the hydrolysis became even slower, and it was complete only after $25 \mathrm{~h}$. The smallest $\mathrm{k}$ values $\left(\mathrm{k}_{1}=0.51 \mathrm{~h}^{-1}, \mathrm{k}_{2}=0.11 \mathrm{~h}^{-1}\right)$ were obtained in this case (Table 2/Entry 2). It is obvious that the lack of the electron-withdrawing $\mathrm{OH}$ group in position $\alpha$, or the appearance of an 
Me group instead of the $\mathrm{HO}$ function decreases the electrophilicity of the $\mathrm{P}$ atom of the $\mathrm{P}=\mathrm{O}$-function. The hydrolysis of $\beta$-phenylethylphosphonate $(4 \mathrm{~m})$ with a reaction time of $20 \mathrm{~h}$ and k values of $0.70 \mathrm{~h}^{-1}$ and $0.15 \mathrm{~h}^{-1}$ (Table 2/Entry 3) occupied an intermediate position.

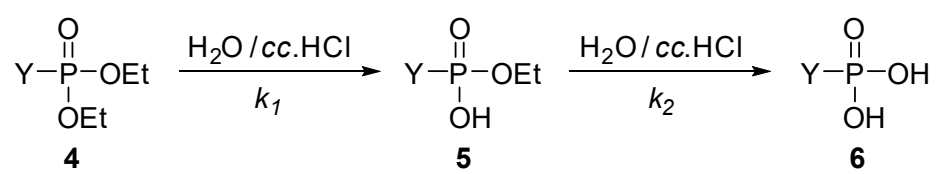

Scheme 2. The two-step hydrolysis of other phosphonate derivatives. For the Y substituents see Table 2.

Table 2. Experimental and kinetic data on the two-step hydrolysis of phosphonates $4 \mathbf{k}-\mathbf{m}$.

\begin{tabular}{ccccccc}
\hline Entry & $\mathbf{Y}$ & $\boldsymbol{t}_{\text {max }}(\mathbf{h})$ & $\mathbf{t}_{\mathbf{r}} \mathbf{( h )}$ & $\mathbf{k}_{\mathbf{1}}\left(\mathbf{h}^{-\mathbf{1}}\right)$ & $\mathbf{k}_{\mathbf{2}}\left(\mathbf{h}^{-\mathbf{1}}\right)$ & $\mathbf{R}^{\mathbf{2}}$ \\
\hline 1 & $\mathrm{PhCH}_{2}(\mathbf{k})$ & 2 & 15 & 1.12 & 0.20 & 0.983 \\
2 & $\mathrm{PhCHMe}(\mathbf{1})$ & 4 & 25 & 0.51 & 0.11 & 0.940 \\
3 & $\begin{array}{c}\mathrm{Ph}\left(\mathrm{CH}_{2}\right)_{2} \\
(\mathbf{m})\end{array}$ & 2.75 & 20 & 0.70 & 0.15 & 0.949 \\
\hline
\end{tabular}

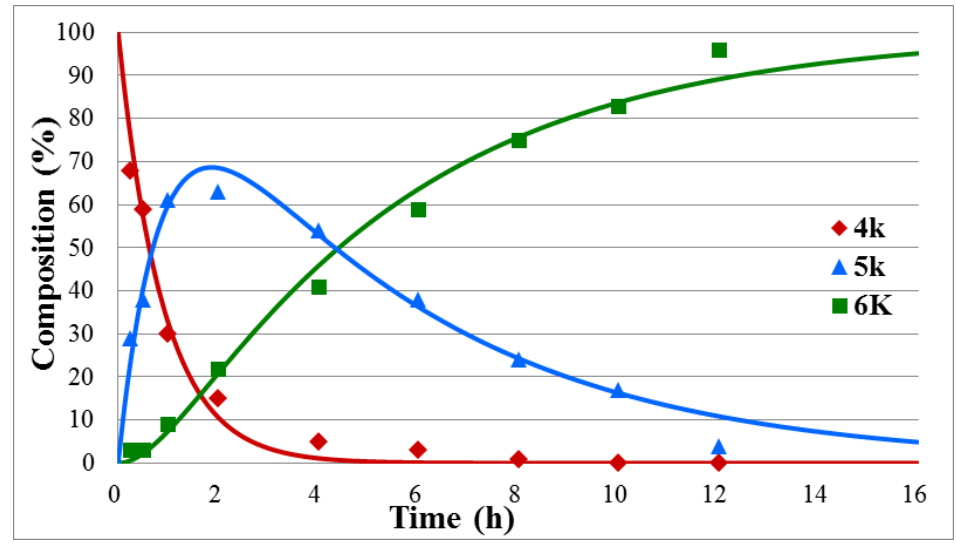

Figure 3. Concentration profile for the components during the hydrolysis of diethyl benzylphosphonate (4k) under optimum conditions. The $\mathrm{R}^{2}$ measure of goodness of fit is 0.983 .

It is noted that the hydrolyses of the phosphonate function take place via the $\mathrm{S}_{\mathrm{N}} 2$ mechanism, i.e., by the nucleophilic attack of the water molecule on the $\mathrm{P}=\mathrm{O}$ function. In the consecutive series, the fission of the second $\mathrm{P}-\mathrm{O}-\mathrm{C}$ bond is the rate-determining step.

The overall order of reactivity of the phosphonates $(\mathbf{1 a}-\mathbf{j}$ and $\mathbf{4 k}-\mathbf{m})$ observed under acidic conditions was summarized in Table 3.

Table 3. Reactivity order of the phosphonates $\mathbf{1 a}-\mathbf{j}$ and $\mathbf{4 k}-\mathbf{m}$ in acidic hydrolyses characterized by $t_{\mathbf{r}}$, as well as $\mathrm{k}_{1}$ and $\mathrm{k}_{2}$.

\begin{tabular}{|c|c|c|c|c|c|c|c|c|}
\hline & $\begin{array}{c}\mathbf{1} \mathbf{b}-\mathbf{d} \\
\mathrm{Y}=\mathrm{NO}_{2}, \mathrm{Cl}\end{array}$ & & $\begin{array}{l}1 \mathrm{~h} \\
\mathrm{Y}=\mathrm{NO}_{2}\end{array}$ & & & & & 41 \\
\hline $\mathrm{t}_{\mathrm{r}}(\mathrm{h})$ & $2.5-6.0$ & 6.5 & $5.5-9.0$ & 8 & 9.5 & 15 & 20 & 25 \\
\hline $\mathrm{k}_{1}\left(\mathrm{~h}^{-1}\right)$ & $3.36-5.18$ & 2.64 & $1.08-1.40$ & 1.64 & 1.03 & 1.12 & 0.70 & 0.51 \\
\hline $\mathrm{k}_{2}\left(\mathrm{~h}^{-1}\right)$ & $0.67-1.24$ & 0.60 & $0.31-0.61$ & 0.31 & 0.35 & 0.20 & 0.15 & 0.11 \\
\hline
\end{tabular}




\section{Materials and Methods}

\subsection{General Information}

The ${ }^{31} \mathrm{P},{ }^{13} \mathrm{C}$, and ${ }^{1} \mathrm{H}$ NMR spectra were taken on a Bruker DRX-500 spectrometer operating at 202.4, 125.7, and $500 \mathrm{MHz}$, respectively. The couplings are given in Hz. LC-MS measurements were performed with an Agilent 1200 liquid chromatography system coupled with a 6130 quadrupole mass spectrometer equipped with an ESI ion source (Agilent Technologies, Palo Alto, CA, USA). High-resolution mass spectrometric measurements were performed using a Thermo Velos Pro Orbitrap Elite hybrid mass spectrometer in positive electrospray mode.

\subsection{Use of the ${ }^{31} P$ NMR Spectra in Quantitative Analysis}

The composition of the reaction mixture was determined by the integration of the areas under the corresponding peaks of the starting material, intermediate, and product in the ${ }^{31} \mathrm{P} \mathrm{NMR}$ spectra.

\subsection{Curve Fitting on the Time-Relative Quantity Data Pairs}

The acidic hydrolysis was modeled assuming pseudo-first-order kinetics. The concentration of water and hydrochloric acid was constant during the reaction, and their initial concentration is incorporated in the pseudo-first-order rate constants $k_{1}$ and $k_{2}$. The corresponding differential equations used in the model are the following:

$$
\begin{gathered}
\frac{d[\text { diester }]}{d t}=-k_{1}[\text { diester }] \\
\frac{d[\text { ester }- \text { acid }]}{d t}=k_{1}[\text { diester }]-k_{2}[\text { ester }- \text { acid }] \\
\frac{d[\text { acid }]}{d t}=k_{2}[\text { ester }- \text { acid }]
\end{gathered}
$$

where [diester], [ester-acid], and [acid] are the time-dependent molarities of the dialkyl $\alpha$ hydroxyphosphonate, the phosphonic ester-acid intermediate, and the phosphonic acid, respectively, and $k_{1}$ and $k_{2}$ are the pseudo-first-order rate constants of the first and the second step of the hydrolysis.

The solution of the differential equations is the following $\left(k_{1} \neq k_{2}\right)$ :

$$
\begin{gathered}
{[\text { diester }]=c_{0} e^{-k_{1} t}} \\
{[\text { ester }- \text { acid }]=c_{0}\left(\frac{k_{1}}{k_{2}-k_{1}} e^{-k_{1} t}-\frac{k_{1}}{k_{2}-k_{1}} e^{-k_{2} t}\right)} \\
{[\text { acid }]=c_{0}\left(1-\frac{k_{2}}{k_{2}-k_{1}} e^{-k_{1} t}+\frac{k_{1}}{k_{2}-k_{1}} e^{-k_{2} t}\right)=c_{0}-[\text { diester }]-[\text { ester }- \text { acid }]}
\end{gathered}
$$

where $c_{0}$ is the initial molarity of the dialkyl $\alpha$-hydroxyphosphonate.

The relative quantity of the components is their molarity divided by the sum of the three molarities (which is $c_{0}$ ). The calculated time-composition curves are described by the following equations, by leaving the $k_{1}$ and $k_{2}$ rate constants as parameters:

$$
\begin{gathered}
\text { diester }_{\text {calcd }}=e^{-k_{1} t} \times 100 \% \\
\text { ester }- \text { acid }_{\text {calcd }}=\frac{k_{1}}{k_{2}-k_{1}}\left(e^{-k_{1} t}-e^{-k_{2} t}\right) \times 100 \% \\
\text { acid }_{\text {calcd }}=100 \%-\text { diester }_{\text {calcd }}-\text { ester }- \text { acid }_{\text {calcd }} .
\end{gathered}
$$


During the calculation of the rate constants, we first gave arbitrary initial values to $k_{1}$ and $k_{2}$, then, we optimized their values such that the sum of the squares of the differences between the experimental and calculated compositions became minimal:

$$
\begin{aligned}
S S_{\text {res }} & =\sum_{i=1}^{n}\left(\text { diester }_{\text {exp }, i}-\operatorname{diester}_{\text {calcd }, i}\right)^{2} \\
& +\sum_{i=1}^{n}\left(\text { ester }-\operatorname{acid}_{\text {exp }, i}-\text { ester }-\operatorname{acid}_{\text {calcd }, i}\right)^{2} \\
& +\sum_{i=1}^{n}\left(\operatorname{acid}_{\text {exp }, i}-\operatorname{acid}_{\text {calcd }, i}\right)^{2} \rightarrow \min
\end{aligned}
$$

where $n$ is the number of experimental time-composition data points measured at reaction times $t_{1}, t_{2}$, $\ldots, t_{n}$.

The resulting $k_{1}$ and $k_{2}$ rate constants and the associated time-composition curves were considered as the best fits. The best fits were found iteratively, using the nonlinear generalized reduced gradient method [19] of Microsoft Excel Solver.

The $\mathrm{R}^{2}$ measure of goodness of fit is calculated as $\mathrm{R}^{2}=1-\left(\frac{S S_{r e s}}{S S_{t o t}}\right)$, where $S S_{r e s}$ is described above and

$$
\begin{aligned}
S S_{t o t} & =\sum_{j=1}^{n}\left(\text { diester }_{\exp , j}-\frac{1}{n} \sum_{i=1}^{n} \operatorname{diester}_{\exp , i}\right)^{2} \\
& +\sum_{i=1}^{n}\left(\operatorname{ester}-\operatorname{acid}_{\exp , j}-\frac{1}{n} \sum_{i=1}^{n}{\text { ester } \left.-\operatorname{acid}_{\text {exp }, i}\right)^{2}}^{2}\right. \\
& +\sum_{j=1}^{n}\left(\operatorname{acid}_{\exp , j}-\frac{1}{n} \sum_{i=1}^{n} \operatorname{acid}_{\exp , j}\right)^{2}
\end{aligned}
$$

The reaction time $\left(t_{\max }\right)$ corresponding to the maximal ratio of the phosphonic ester-acid intermediate in the reaction mixture was found as follows:

$$
\begin{gathered}
0=\left.\frac{d\left[\text { ester }- \text { acid }_{\text {calcd }}\right]}{d t}\right|_{t_{\max }}=\left.\frac{d}{d t}\left(\frac{k_{1}}{k_{2}-k_{1}}\left(e^{-k_{1} t}-e^{-k_{2} t}\right) \times 100 \%\right)\right|_{t_{\max }} \\
0=\left.\frac{d}{d t}\left(e^{-k_{1} t}-e^{-k_{2} t}\right)\right|_{t_{\max }}=-k_{1} e^{-k_{1} t_{\max }}+k_{2} e^{-k_{2} t_{\max }} \\
k_{1} e^{-k_{1} t_{\max }}=k_{2} e^{-k_{2} t_{\max }} \\
\frac{k_{1}}{k_{2}}=\frac{e^{-k_{2} t_{\max }}}{e^{-k_{1} t_{\max }}} \equiv e^{\left(k_{1}-k_{2}\right) t_{\max }} \\
t_{\max }=\frac{\ln \left(\frac{k_{1}}{k_{2}}\right)}{k_{1}-k_{2}} .
\end{gathered}
$$

\subsection{General Procedure for the Hydrolysis of Phosphonates (1a-j, $\mathbf{4 k}-\mathbf{m})$}

A mixture of $3.8 \mathrm{mmol}$ of phosphonate $(\mathbf{1 a}: 0.82 \mathrm{~g}, \mathbf{1 b}: 0.99 \mathrm{~g}, \mathbf{1 c}: 0.95 \mathrm{~g}, \mathbf{1 d}: 0.89 \mathrm{~g}, \mathbf{1 e}: 1.1 \mathrm{~g}, \mathbf{1 f}$ : 0.87 g, 1g: 0.93 g, 1h: 1.1 g, 1i: 1.1 g, 1j: 1.0 g, 4k: 0.87 g, $41: 0.92$ g, 4m: 0.92 g), $1.0 \mathrm{~mL}(6.0 \mathrm{mmol})$ of cc. hydrochloric acid, and $2.0 \mathrm{~mL}$ of water was stirred at reflux for $2.5-25 \mathrm{~h}$. The concentration of an aliquot part of the reaction mixture, or the whole mixture, afforded an oil that was analyzed by ${ }^{31} \mathrm{P}$ NMR spectroscopy and LC-MS. Identification of the starting materials $(\mathbf{1} \mathbf{a}-\mathbf{j}, \mathbf{4} \mathbf{k}-\mathbf{m})$, intermediates $(\mathbf{2} \mathbf{a}-\mathbf{j}, \mathbf{5 k}-\mathbf{m})$, and products $(\mathbf{3 A}-\mathbf{F}, \mathbf{6 K}-\mathbf{M})$ can be found in Table 4 . The ${ }^{13} \mathrm{C}$ and ${ }^{1} \mathrm{H}$ NMR spectral data of the new intermediates $(\mathbf{2} \mathbf{a}-\mathbf{f}, \mathbf{h}-\mathbf{j})$ were obtained from the spectra of the corresponding mixtures containing also the phosphonic acids (3A-F). 
Table 4. Identification of the starting phosphonates $(\mathbf{1} \mathbf{a}-\mathbf{j}, \mathbf{4 k}-\mathbf{m})$, ester-acid intermediates $(\mathbf{2} \mathbf{a}-\mathbf{j}, \mathbf{5 k}-\mathbf{m})$, and phosphonic acids (3A-F, 6K-M)

\begin{tabular}{|c|c|c|c|}
\hline & \multicolumn{2}{|c|}{$\delta^{31}$ P NMR } & \multirow{2}{*}[\mathbf{M}+\mathbf{H}]{} \\
\hline & Found (DMSO) & Literature & \\
\hline 1a & 23.9 & $23.9\left(\mathrm{CDCl}_{3}\right)[20]$ & 216.9 \\
\hline $1 b$ & 22.7 & $21.9\left(\mathrm{CDCl}_{3}\right)[20]$ & 262.2 \\
\hline 1c & 23.5 & $22.1\left(\mathrm{CDCl}_{3}\right)[20]$ & 273.0 \\
\hline 1d & 23.8 & $23.3\left(\mathrm{CDCl}_{3}\right)[21]$ & 235.0 \\
\hline $\mathbf{1 e}[22]^{\mathrm{a}}$ & 23.1 & - & 285.1 \\
\hline 1f & 24.1 & $24.0\left(\mathrm{CDCl}_{3}\right)[20]$ & 231 \\
\hline $1 \mathrm{~g}$ & 21.8 & $21.5\left(\mathrm{CDCl}_{3}\right)[20]$ & 245.1 \\
\hline $1 \mathrm{~h}$ & 20.4 & $19.9\left(\mathrm{CDCl}_{3}\right)[20]$ & 290.1 \\
\hline $\mathbf{1 i}$ & 21.2 & $21.9\left(\mathrm{CDCl}_{3}\right)[20]$ & 354.1 \\
\hline $1 \mathrm{j}$ & 21.7 & $21.7\left(\mathrm{CDCl}_{3}\right)[23]$ & 262.1 \\
\hline $4 \mathrm{k}$ & 26.7 & $26.4\left(\mathrm{CDCl}_{3}\right)[24]$ & 229.2 \\
\hline 41 & 29.8 & $30.4\left(\mathrm{CDCl}_{3}\right)[25]$ & 243.1 \\
\hline $4 m$ & 30.6 & $30.8\left(\mathrm{CDCl}_{3}\right)[24]$ & 243.1 \\
\hline $2 a$ & 20.8 & $19.9\left(\mathrm{D}_{2} \mathrm{O}\right)[26]$ & $203.04675\left(203.04677^{c}\right)$ \\
\hline $2 b^{b}$ & 18.8 & & $248.03164\left(248.03185^{c}\right)$ \\
\hline $2 c^{b}$ & 20.3 & & $237.00752\left(237.00780^{c}\right)$ \\
\hline $2 d^{b}$ & 20.4 & & $221.03692\left(221.03735^{c}\right)$ \\
\hline $2 e^{b}$ & 19.6 & & $271.03384\left(271.03416^{c}\right)$ \\
\hline $2 f^{b}$ & 20.8 & & $217.06177\left(217.06242^{c}\right)$ \\
\hline $2 \mathbf{g}[27]^{a}$ & 19.6 & & $217.06193\left(217.06242^{c}\right)$ \\
\hline $2 h^{b}$ & 18.1 & & $262.04707\left(262.04750^{c}\right)$ \\
\hline $2 \mathbf{i}^{b}$ & 19.0 & & $251.02306\left(251.02345^{\mathrm{c}}\right)$ \\
\hline $2 j^{b}$ & 19.5 & & $235.05267\left(235.05300^{c}\right)$ \\
\hline $5 k$ & 23.7 & $25.5\left(\mathrm{CD}_{3} \mathrm{OD}\right)[28]$ & 201.1 \\
\hline 51 & 27.0 & $32.3\left(\mathrm{CDCl}_{3}\right)[29]$ & 215.1 \\
\hline $5 \mathrm{~m}[30]^{\mathrm{a}}$ & 27.5 & - & 215.1 \\
\hline $3 \mathrm{~A}$ & 18.9 & $19.4\left(\mathrm{D}_{2} \mathrm{O}\right)[18]$ & 189.1 \\
\hline 3B & 16.6 & $16.4\left(\mathrm{D}_{2} \mathrm{O}\right)[18]$ & 234.0 \\
\hline $3 C$ & 17.9 & $18.6\left(\mathrm{D}_{2} \mathrm{O}\right)[18]$ & 223.0 \\
\hline $3 \mathrm{D}$ & 18.2 & $18.5[31]$ & 207.0 \\
\hline $3 E^{b}$ & 17.4 & & 257.0 \\
\hline $3 F$ & 18.6 & $18.8\left(\mathrm{D}_{2} \mathrm{O}\right)[18]$ & 203.1 \\
\hline $6 K$ & 21.8 & 22.1 (DMSO) [32] & 173.0 \\
\hline $6 \mathrm{~L}$ & 25.7 & $27.8\left(\mathrm{CD}_{3} \mathrm{OD}\right)[33]$ & 187.0 \\
\hline $6 \mathrm{M}$ & 26.0 & 26.7 (DMSO) [34] & 187.0 \\
\hline
\end{tabular}

${ }^{\text {a } 13} \mathrm{C}$ and ${ }^{1} \mathrm{H}$ NMR characterization is available in the literature; ${ }^{\mathrm{b}}$ Spectral data are given below; ${ }^{\mathrm{c}}$ Calculated value.

${ }^{13} \mathrm{C}$ and ${ }^{1} \mathrm{H}$ NMR characterization of the new ester acids:

Methyl hydrogen 1-(4-nitrophenyl)-1-hydroxymethylphosphonate (2b). ${ }^{13} \mathrm{C}$ NMR (DMSO-d $\left.d_{6}\right) \delta: 52.6\left(\mathrm{~d},{ }^{2} J=\right.$ 6.5, $\left.\mathrm{OCH}_{3}\right), 69.1\left(\mathrm{~d},{ }^{1} J=157.5, \mathrm{PCH}\right), 122.7\left(\mathrm{~d},{ }^{4} J=2.4, \mathrm{C}_{3}\right), 128.1\left(\mathrm{~d},{ }^{3} J=5.0, \mathrm{C}_{2}\right), 146.5\left(\mathrm{~d},{ }^{5} J=3.3, \mathrm{C}_{4}\right)$, $147.6\left(\mathrm{C}_{1}\right) ;{ }^{1} \mathrm{H}$ NMR (DMSO $\left.d_{6}\right) \delta: 3.58\left(\mathrm{~d},{ }^{3} \mathrm{~J}=10.4,3 \mathrm{H}, \mathrm{OMe}\right), 5.05\left(\mathrm{~d},{ }^{2} \mathrm{~J}=15.5,1 \mathrm{H}, \mathrm{PCH}\right), 7.64-7.73$ $\left(\mathrm{m}, 2 \mathrm{H}, \mathrm{H}_{2}\right), 8.17-8.23\left(\mathrm{~m}, 2 \mathrm{H}, \mathrm{H}_{3}\right)$.

Methyl hydrogen 1-(4-chlorophenyl)-1-hydroxymethylphosphonate (2c). ${ }^{13} \mathrm{C}$ NMR (DMSO- $\left.d_{6}\right) \delta: 53.0$ (d, $\left.{ }^{2} J=6.5, \mathrm{OCH}_{3}\right), 69.4\left(\mathrm{~d},{ }^{1} J=160.3, \mathrm{PCH}\right), 128.1\left(\mathrm{~d},{ }^{4} J=2.2, \mathrm{C}_{3}\right), 129.5\left(\mathrm{~d},{ }^{3} J=5.3, \mathrm{C}_{2}\right), 132.1\left(\mathrm{~d},{ }^{5} \mathrm{~J}=\right.$ 3.6, $\left.\mathrm{C}_{4}\right), 139.0\left(\mathrm{C}_{1}\right) ;{ }^{1} \mathrm{H}$ NMR (DMSO- $\left.d_{6}\right) \delta: 3.55\left(\mathrm{~d},{ }^{3} \mathrm{~J}=10.3,3 \mathrm{H}, \mathrm{OMe}\right), 4.85\left(\mathrm{~d},{ }^{2} J=13.8,1 \mathrm{H}, \mathrm{PCH}\right)$, 7.32-7.47 (m, 4H, Ar).

Methyl hydrogen 1-(4-fluorophenyl)-1-hydroxymethylphosphonate (2d). ${ }^{13} \mathrm{C}$ NMR (DMSO- $d_{6}$ ) $8: 53.0$ (d, $\left.{ }^{2} J_{\mathrm{P}, \mathrm{C}}=6.5, \mathrm{OCH}_{3}\right), 69.4\left(\mathrm{~d},{ }^{1} J_{\mathrm{P}, \mathrm{C}}=161.3, \mathrm{PCH}\right), 114.9\left(\mathrm{dd},{ }^{2} J_{\mathrm{F}, \mathrm{C}}=21.2,{ }^{4} J_{\mathrm{P}, \mathrm{C}}=1.9, \mathrm{C}_{3}\right), 129.7\left(\mathrm{dd},{ }^{3} J_{\mathrm{F}, \mathrm{C}}=\right.$ $\left.8.0,{ }^{3} J_{\mathrm{P}, \mathrm{C}}=5.6, \mathrm{C}_{2}\right), 136.1\left(\mathrm{~d},{ }^{4} J_{\mathrm{F}, \mathrm{C}}=2.6\right), 161.9\left(\mathrm{dd},{ }^{1} J_{\mathrm{F}, \mathrm{C}}=242.5,{ }^{5} J_{\mathrm{P}, \mathrm{C}}=3.0, \mathrm{C}_{4}\right) ;{ }^{1} \mathrm{H}$ NMR $\left(\mathrm{DMSO}-d_{6}\right) \delta$ : $3.54\left(\mathrm{~d},{ }^{3} \mathrm{~J}=10.2,3 \mathrm{H}, \mathrm{OMe}\right), 4.84\left(\mathrm{~d},{ }^{2} \mathrm{~J}=13.2,1 \mathrm{H}, \mathrm{PCH}\right), 7.09-7.19\left(\mathrm{~m}, 2 \mathrm{H}, \mathrm{H}_{3}\right), 7.38-7.49\left(\mathrm{~m}, 2 \mathrm{H}, \mathrm{H}_{2}\right)$. 
Methyl hydrogen 1-(4-trifluoromethylphenyl)-1-hydroxymethylphosphonate (2e). ${ }^{13} \mathrm{C}$ NMR $\left(\mathrm{DMSO}-d_{6}\right) \delta$ : $53.1\left(\mathrm{~d},{ }^{2} J_{\mathrm{P}, \mathrm{C}}=6.5, \mathrm{OCH}_{3}\right), 69.7\left(\mathrm{~d},{ }^{1} J_{\mathrm{P}, \mathrm{C}}=157.9, \mathrm{PCH}\right), 124.7-125.0\left(\mathrm{~m}, \mathrm{C}_{3}\right), 124.9\left(\mathrm{q},{ }^{1} J_{\mathrm{F}, \mathrm{C}}=271.1, \mathrm{CF}_{3}\right)$, 127.3-128.7 (m, $\left.\mathrm{C}_{2}, \mathrm{C}_{4}\right), 145.0\left(\mathrm{C}_{1}\right) ;{ }^{1} \mathrm{H}$ NMR $\left(\mathrm{DMSO}-\mathrm{d}_{6}\right) \delta: 3.56\left(\mathrm{~d},{ }^{3} \mathrm{~J}=10.3,3 \mathrm{H}, \mathrm{OMe}\right), 4.96\left(\mathrm{~d},{ }^{2} \mathrm{~J}=\right.$ 14.6, 1H, PCH), 7.57-7.72 (m, 4H, Ar).

Methyl hydrogen 1-(4-methylphenyl)-1-hydroxymethylphosphonate (2f). ${ }^{13} \mathrm{C}$ NMR (DMSO- $\left.d_{6}\right) \delta: 21.2$ $\left(\mathrm{Ar}_{-} \mathrm{CH}_{3}\right), 52.8\left(\mathrm{~d},{ }^{2} J_{\mathrm{P}, \mathrm{C}}=6.6, \mathrm{OCH}_{3}\right), 70.0\left(\mathrm{~d},{ }^{1} J_{\mathrm{P}, \mathrm{C}}=160.2, \mathrm{PCH}\right), 127.7\left(\mathrm{~d},{ }^{3} \mathrm{~J}=5.6, \mathrm{C}_{2}\right), 128.7\left(\mathrm{~d},{ }^{4} \mathrm{~J}=\right.$ $\left.2.1, \mathrm{C}_{3}\right), 136.5\left(\mathrm{~d},{ }^{5} \mathrm{~J}=3.1, \mathrm{C}_{4}\right), 136.9\left(\mathrm{C}_{1}\right),{ }^{1} \mathrm{H}$ NMR $\left(\mathrm{DMSO}-\mathrm{d}_{6}\right) \delta: 2.28\left(\mathrm{~s}, 3 \mathrm{H}, \mathrm{Ar}-\mathrm{CH}_{3}\right), 3.52\left(\mathrm{~d},{ }^{3} \mathrm{~J}=10.2\right.$, $3 \mathrm{H}, \mathrm{OMe}), 4.76\left(\mathrm{~d},{ }^{2} \mathrm{~J}=13.0,1 \mathrm{H}, \mathrm{PCH}\right), 7.06-7.38(\mathrm{~m}, 4 \mathrm{H}, \mathrm{Ar})$.

Ethyl hydrogen 1-(4-nitrophenyl)-1-hydroxymethylphosphonate $(2 \mathrm{~h}){ }^{13} \mathrm{C} \mathrm{NMR}\left(\mathrm{DMSO}-\mathrm{d}_{6}\right) \delta: 16.9\left(\mathrm{~d},{ }^{3} \mathrm{~J}=\right.$ 5.6, $\left.\mathrm{CH}_{3}\right), 62.0\left(\mathrm{~d},{ }^{2} \mathrm{~J}=6.5, \mathrm{OCH}_{2}\right), 69.1\left(\mathrm{~d},{ }^{1} \mathrm{~J}=157.5, \mathrm{PCH}\right), 122.7\left(\mathrm{~d},{ }^{4} \mathrm{~J}=2.4, \mathrm{C}_{3}\right), 128.2\left(\mathrm{~d},{ }^{3} \mathrm{~J}=4.9, \mathrm{C}_{2}\right)$, $146.5\left(\mathrm{~d},{ }^{5} \mathrm{~J}=3.6, \mathrm{C}_{4}\right), 147.6\left(\mathrm{C}_{1}\right) ;{ }^{1} \mathrm{H}$ NMR $\left(\mathrm{DMSO}-\mathrm{d}_{6}\right) \delta: 1.17\left(\mathrm{t},{ }^{3} \mathrm{~J}_{\mathrm{H}, \mathrm{H}}=7.0,3 \mathrm{H}, \mathrm{CH}_{3}\right), 3.91\left(\mathrm{dq},{ }^{3} J_{\mathrm{P}, \mathrm{H}}=\right.$ $\left.8.1,{ }^{3} J_{\mathrm{H}, \mathrm{H}}=7.0,2 \mathrm{H}, \mathrm{OCH}_{2}\right), 5.03\left(\mathrm{~d},{ }^{2} \mathrm{~J}=15.7,1 \mathrm{H}, \mathrm{PCH}\right), 7.65-7.72\left(\mathrm{~m}, 2 \mathrm{H}, \mathrm{H}_{2}\right), 8.16-8.23\left(\mathrm{~m}, 2 \mathrm{H}, \mathrm{H}_{3}\right)$. Ethyl hydrogen 1-(4-chlorophenyl)-1-hydroxymethylphosphonate (2i). ${ }^{13} \mathrm{C}$ NMR (DMSO- $\left.d_{6}\right) \delta: 16.6\left(\mathrm{~d},{ }^{3} \mathrm{~J}=\right.$ $\left.5.5, \mathrm{CH}_{3}\right), 61.7\left(\mathrm{~d},{ }^{2} \mathrm{~J}=6.5, \mathrm{OCH}_{2}\right), 69.3\left(\mathrm{~d},{ }^{1} \mathrm{~J}=160.7, \mathrm{PCH}\right), 127.7\left(\mathrm{~d},{ }^{4} \mathrm{~J}=1.9, \mathrm{C}_{3}\right), 129.2\left(\mathrm{~d},{ }^{3} \mathrm{~J}=5.3, \mathrm{C}_{2}\right)$, $131.8\left(\mathrm{~d},{ }^{5} \mathrm{~J}=3.7, \mathrm{C}_{4}\right), 138.6\left(\mathrm{C}_{1}\right) ;{ }^{1} \mathrm{H}$ NMR $\left(\mathrm{DMSO}-\mathrm{d}_{6}\right) \delta: 1.15\left(\mathrm{t},{ }^{3} \mathrm{~J}_{\mathrm{H}, \mathrm{H}}=7.1,3 \mathrm{H}, \mathrm{CH}_{3}\right), 3.91\left(\mathrm{dq},{ }^{3} J_{\mathrm{P}, \mathrm{H}}=\right.$ $\left.7.1,{ }^{3} \mathrm{~J}_{\mathrm{H}, \mathrm{H}}=7.1,2 \mathrm{H}, \mathrm{OCH}_{2}\right), 4.84\left(\mathrm{~d},{ }^{2} \mathrm{~J}=13.8,1 \mathrm{H}, \mathrm{PCH}\right), 7.33-7.47(\mathrm{~m}, 4 \mathrm{H}, \mathrm{Ar})$.

Ethyl hydrogen 1-(4-fluorophenyl)-1-hydroxymethylphosphonate (2j). ${ }^{13} \mathrm{C}$ NMR (DMSO- $\left.d_{6}\right) \delta: 16.9\left(\mathrm{~d},{ }^{3} \mathrm{~J}=\right.$ 5.6, $\left.\mathrm{CH}_{3}\right), 61.9\left(\mathrm{~d},{ }^{2} \mathrm{~J}=6.5, \mathrm{OCH}_{2}\right), 69.5\left(\mathrm{~d},{ }^{1} J_{\mathrm{P}, \mathrm{C}}=161.7, \mathrm{PCH}\right), 114.8\left(\mathrm{dd},{ }^{2} J_{\mathrm{F}, \mathrm{C}}=21.1,{ }^{4} J_{\mathrm{P}, \mathrm{C}}=2.0, \mathrm{C}_{3}\right)$, $129.7\left(\mathrm{dd},{ }^{3} J_{\mathrm{F}, \mathrm{C}}=8.2,{ }^{3} J_{\mathrm{P}, \mathrm{C}}=5.5, \mathrm{C}_{2}\right), 136.1\left(\mathrm{~d},{ }^{4} J_{\mathrm{F}, \mathrm{C}}=2.8\right), 161.9\left(\mathrm{dd},{ }^{1} J_{\mathrm{F}, \mathrm{C}}=242.4,{ }^{5} J_{\mathrm{P}, \mathrm{C}}=3.2, \mathrm{C}_{4}\right) ;{ }^{1} \mathrm{H}$ NMR (DMSO-d $\left.d_{6}\right) \delta: 1.15\left(\mathrm{t}^{3} \mathrm{~J}_{\mathrm{H}, \mathrm{H}}=7.0,3 \mathrm{H}, \mathrm{CH}_{3}\right), 3.87-3.95\left(\mathrm{~m}, 2 \mathrm{H}, \mathrm{OCH}_{2}\right), 4.82\left(\mathrm{~d},{ }^{2} \mathrm{~J}=13.3,1 \mathrm{H}, \mathrm{PCH}\right)$, 7.11-7.17 (m, 2H, $\left.\mathrm{H}_{3}\right), 7.42-7.47\left(\mathrm{~m}, 2 \mathrm{H}, \mathrm{H}_{2}\right)$.

1-(4-Trifluoromethylphenyl)-1-hydroxymethylphosphonic acid (3e). ${ }^{13} \mathrm{C}$ NMR (DMSO-d 6$) \delta: 70.5\left(\mathrm{~d},{ }^{1} J_{\mathrm{P}, \mathrm{C}}=\right.$ 157.7, PCH), 124.7-125.0 (m, $\left.\mathrm{C}_{3}\right), 124.9\left(\mathrm{q},{ }^{1} J_{\mathrm{F}, \mathrm{C}}=272.3, \mathrm{CF}_{3}\right), 127.2-128.6\left(\mathrm{~m}, \mathrm{C}_{4}\right), 128.4\left(\mathrm{~d},{ }^{3} J_{\mathrm{P}, \mathrm{C}}=5.0\right.$, $\left.\mathrm{C}_{2}\right), 145.6\left(\mathrm{C}_{1}\right) ;{ }^{1} \mathrm{H}$ NMR (DMSO-d 6$) \delta: 4.80\left(\mathrm{~d},{ }^{2} \mathrm{~J}=15.0,1 \mathrm{H}, \mathrm{PCH}\right), 7.55-7.72(\mathrm{~m}, 4 \mathrm{H}, \mathrm{Ar})$.

\section{Conclusions}

Kinetic study of the two-step acidic hydrolysis of a series of dialkyl $\alpha$-hydroxybenzylphosphonates and a few related model compounds allowed the mapping of the reactivity of the different substrates. The two-step hydrolyses were characterized by $\mathrm{k}_{1}$ and $\mathrm{k}_{2}$ pseudo-first-order rate constants belonging to the formation of the corresponding monoester monoacids and the phosphonic acids, respectively. Electron-withdrawing substituents increased the rate, while electron-releasing ones slowed down the hydrolyses starting with the nucleophilic attack of the water molecule. It turned out that the fission of the second $\mathrm{P}-\mathrm{O}-\mathrm{C}$ unit is the rate-determining step. The intermediate ester-acid species were identified and characterized.

Supplementary Materials: The following are available online: Figure S1: Concentration profile for the components during the hydrolysis of dimethyl $\alpha$-hydroxy-4-nitrobenzylphosphonate (1b) under optimum conditions. The $\mathrm{R}^{2}$ measure of goodness of fit is 0.989 . Figure S2: Concentration profile for the components during the hydrolysis of dimethyl $\alpha$-hydroxy-4-chlorobenzylphosphonate (1c) under optimum conditions. The $\mathrm{R}^{2}$ measure of goodness of fit is 0.987 . Figure S3: Concentration profile for the components during the hydrolysis of dimethyl $\alpha$-hydroxy-4-fluorobenzylphosphonate (1d) under optimum conditions. The $R^{2}$ measure of goodness of fit is 0.965 . Figure S4: Concentration profile for the components during the hydrolysis of dimethyl $\alpha$-hydroxy-4-trifluoromethylbenzylphosphonate (1e) under optimum conditions. The $\mathrm{R}^{2}$ measure of goodness of fit is 0.988 . Figure S5: Concentration profile for the components during the hydrolysis of dimethyl $\alpha$-hydroxy-4-methylbenzylphosphonate (1f) under optimum conditions. The $\mathrm{R}^{2}$ measure of goodness of fit is 0.962. Figure S6: Concentration profile for the components during the hydrolysis of diethyl $\alpha$-hydroxy-4-nitrobenzylphosphonate (1h) under optimum conditions. The $\mathrm{R}^{2}$ measure of goodness of fit is 0.992. Figure S7: Concentration profile for the components during the hydrolysis of diethyl $\alpha$-hydroxy-4-chlorobenzylphosphonate (1i) under optimum conditions. The $\mathrm{R}^{2}$ measure of goodness of fit is 0.992. Figure S8: Concentration profile for the components during the hydrolysis of diethyl $\alpha$-hydroxy-4-fluorobenzylphosphonate (1j) under optimum conditions. The $R^{2}$ measure of goodness of fit is 0.970 . Figure S9: Concentration profile for the components during the hydrolysis of diethyl $\alpha$-phenylethylphosphonate (41) under optimum conditions. The $\mathrm{R}^{2}$ measure of goodness of fit is 0.940. Figure S10: Concentration profile for the 
components during the hydrolysis of diethyl $\beta$-phenylethylphosphonate (4m) under optimum conditions. The $\mathrm{R}^{2}$ measure of goodness of fit is 0.949 .

Author Contributions: Conceptualization, G.K. and Z.R.; methodology, N.H.; software, Á.S.; formal analysis, N.H. and J.K.; investigation, N.H.; resources, G.K.; data curation, Á.S./N.H.; writing-original draft preparation, G.K. and N.H.; writing-review and editing, G.K.; supervision, G.K.; project administration, G.K.; funding acquisition, G.K. All authors have read and agreed to the published version of the manuscript.

Funding: This project was funded by the National Research, Development and Innovation Office (K119202 and K134318).

Conflicts of Interest: The authors declare no conflict of interest.

\section{References}

1. Desai, J.; Wang, Y.; Wang, K.; Malwal, S.R.; Oldfield, E. Isoprenoid biosynthesis inhibitors targeting bacterial cell growth. Chem. Med. Chem. 2016, 11, 2205-2215. [CrossRef] [PubMed]

2. Tcarkova, K.V.; Artyushin, O.I.; Bondarenko, N.A. Synthetic routes to bis(3-aminophenyl) phosphinic acid. Phosphorus Sulfur Silicon 2016, 191, 1520-1522. [CrossRef]

3. Keglevich, G.; Grün, A.; Bölcskei, A.; Drahos, L.; Kraszni, M.; Balogh, G.T. Synthesis and proton dissociation properties of arylphosphonates; A microwave-assisted catalytic Arbuzov reaction with aryl bromides. Heteroat. Chem. 2012, 23, 574-582. [CrossRef]

4. Gavande, N.; Yamamoto, I.; Salam, N.K.; Ai, T.-H.; Burden, P.M.; Johnston, G.A.R.; Hanrahan, J.R.; Chebib, M. Novel cyclic phosphinic acids as $\mathrm{GABA}_{\mathrm{C}} \rho$ receptor antagonists: Design, synthesis, and pharmacology. ACS Med. Chem. Lett. 2011, 2, 11-16. [CrossRef] [PubMed]

5. Rahil, J.; Haake, P. Rates and mechanism of the alkaline-hydrolysis of a sterically hindered phosphinate ester-Partial reaction by nucleophilic-attack at carbon. J. Org. Chem. 1981, 46, 3048-3052. [CrossRef]

6. Cook, R.D.; Farah, S.; Ghawi, L.; Itani, A.; Rahil, J. The influence of the changing of $\mathrm{P}=\mathrm{O}$ to $\mathrm{P}=\mathrm{S}$ and $\mathrm{P}-\mathrm{O}-\mathrm{R}$ to P-S-R on the reactivity of phosphinate esters under alkaline-hydrolysis conditions. Can. J. Chem. 1986, 64, 1630-1637. [CrossRef]

7. Wróblewski, A.E.; Verkade, J.G. 1-oxo-2-oxa-1-phosphabicyclo [2.2.2]octane: A new mechanistic probe for the basic hydrolysis of phosphate esters. J. Am. Chem. Soc. 1996, 118, 10168-10174. [CrossRef]

8. Cevasco, G.; Thea, S. The quest for carbanion-promoted dissociative pathways in the hydrolysis of aryl phosphinates. J. Chem. Soc. Perkin Trans. 2 1993, 1103-1106. [CrossRef]

9. Salomon, C.J.; Breuer, E. Efficient and selective dealkylation of phosphonate diisopropyl esters using $\mathrm{ME}_{3} \mathrm{SiBr}$. Tetrahedron Lett. 1995, 36, 6759-6760. [CrossRef]

10. Tulsi, N.S.; Downey, A.M.; Cairo, C.W. A protected L-bromophosphonomethylphenylalanine amino acid derivative (BrPmp) for synthesis of irreversible protein tyrosine phosphatase inhibitors. Bioorg. Med. Chem. 2010, 18, 8679-8686. [CrossRef]

11. Jansa, P.; Hradil, O.; Baszczyňski, O.; Dračínský, M.; Janeba, Z. An efficient microwave-assisted synthesis and biological properties of polysubstituted pyrimidinyl- and 1,3,5-triazinylphosphonic acids. Tetrahedron 2012, 68, 865-871. [CrossRef] [PubMed]

12. Keglevich, G.; Rádai, Z.; Harsági, N.; Szigetvári, Á.; Kiss, N.Z. A study on the acidic hydrolysis of cyclic phosphinates: 1-Alkoxy-3-phospholene 1-oxides, 1-ethoxy-3-methylphospholane 1-oxide, and 1-ethoxy-3-methyl-1,2,3,4,5,6-hexahydrophosphinine 1-oxide. Heteroat. Chem. 2017, 28, e21394. [CrossRef]

13. Harsági, N.; Rádai, Z.; Kiss, N.Z.; Szigetvári, Á.; Keglevich, G. Two-step acidic hydrolysis of dialkyl arylhosphonates. Mendeleev Commun. 2020, 30, 38-39. [CrossRef]

14. Rádai, Z.; Keglevich, G. Synthesis and reactions of $\alpha$-hydroxyphosphonates. Molecules 2018, $23,1493$. [CrossRef] [PubMed]

15. Kiss, N.Z.; Rádai, Z.; Mucsi, Z.; Keglevich, G. Synthesis of aminophosphonates from $\alpha$-hydroxyphosphonates; a theoretical study. Heteroat. Chem. 2016, 27, 260-268. [CrossRef]

16. Rádai, Z.; Hodula, V.; Kiss, N.Z.; Kóti, J.; Keglevich, G. Phosphorylation of (1-aryl-1-hydroxymethyl)phosphonates. Mendeleev Commun. 2019, 29, 153-154. [CrossRef]

17. Rádai, Z.; Szabó, R.; Szigetvári, Á.; Kiss, N.Z.; Mucsi, Z.; Keglevich, G. A study on the rearrangement of dialkyl 1-aryl-1-hydroxymethylphosphonates to benzyl phosphates. Curr. Org. Chem. 2020, 24, 465-471. [CrossRef] 
18. Rádai, Z.; Szeles, P.; Kiss, N.Z.; Hegedûs, L.; Windt, T.; Nagy, V.; Keglevich, G. Green synthesis and cytotoxic activity of dibenzyl $\alpha$-hydroxyphosphonates and $\alpha$-hydroxyphosphonic acids. Heteroat. Chem. 2018, 29, e21436. [CrossRef]

19. Lasdon, L.S.; Waren, A.D.; Jain, A.; Ratner, M. Design and testing of a generalized reduced gradient code for nonlinear programming. ACM T Math. Softw. 1978, 4, 34-50. [CrossRef]

20. Keglevich, G.; Tóth, V.R.; Drahos, L. Microwave-assisted synthesis of $\alpha$-hydroxy-benzylphosphonates and -benzylphosphine oxides. Heteroat. Chem. 2011, 22, 15-17. [CrossRef]

21. Seven, O.; Polat-Cakir, S.; Hossain, M.S.; Emrullahoglu, M. Reactions of acyl phosphonates with organoaluminum reagents: A new method for the synthesis of secondary and tertiary alpha-hydroxy phosphonates. Tetrahedron 2011, 67, 3464-3469. [CrossRef]

22. Cai, Z.-H.; Du, G.-F.; He, L.; Gu, C.-Z.; Dai, B. N-Heterocyclic carbene catalyzed hydrophosphonylation of aldehydes. Synthesis 2011, 2073-2078. [CrossRef]

23. de Noronha, R.G.; Costa, P.J.; Romano, C.C.; Calhorda, M.J.; Fernandes, A.C. $\mathrm{MoO}_{2} \mathrm{Cl}_{2}$ as a novel catalyst for C-P bond formation and for hydrophosphonylation of aldehydes. Organometallics 2009, 28, 6206-6212. [CrossRef]

24. Kedrowski, S.M.A.; Dougherty, D.A. Room-temperature alternative to the Arbuzov reaction: The reductive deoxygenation of acyl phosphonates. Org. Lett. 2010, 12, 3990-3993. [CrossRef]

25. Huang, T.; Chen, T.; Han, L.-B. Oxidative dephosphorylation of benzylic phosphonates with dioxygen generating symmetrical trans-stilbenes. J. Org. Chem. 2018, 83, 2959-2965. [CrossRef]

26. St. Maurice, M.; Bearne, S.L. Reaction intermediate analogues for mandelate racemase: Interaction between Asn 197 and the $\alpha$-hydroxyl of the substrate promotes catalysis. Biochemistry 2000, 39, 13324-13335. [CrossRef]

27. Hamerschmidt, F.; Hanninger, A. Enantioselective deprotonation of benzyl phosphates by homochiral lithium amide bases-Configurational stability of benzyl carbanions with a dialkoxyphosphoryloxy substituent and their rearrangement to optically-active $\alpha$-hydroxy phosphonates. Chem. Ber. 1995, 128, 823-830. [CrossRef]

28. Mortier, J.; Gridnev, I.D.; Guénot, P. Reactions of phosphonates with organohaloboranes: New route to molecular borophosphonates. Organometallics 2000, 19, 4266-4275. [CrossRef]

29. Eom, D.; Jeong, Y.; Kim, Y.R.; Lee, E.; Choi, W.; Lee, P.H. Palladium-catalyzed C( $\mathrm{sp}^{2}$ and sp $\left.{ }^{3}\right)-H$ activation/C-O bond formation: Synthesis of benzoxaphosphole 1- and 2-oxides. Org. Lett. 2013, 15, 5210-5213. [CrossRef]

30. Galardy, R.E.; Kontoyiannidou-Ostrem, V.; Kortylewicz, Z.P. Inhibition of angiotensin converting enzyme by phosphonic amides and phosphonic-acids. Biochemistry 1983, 22, 1990-1995. [CrossRef]

31. Prishchenko, A.A.; Livantsov, M.V.; Novikova, O.P.; Livantsova, L.I.; Maryashkin, A.V. Reaction of trimethylsilyl phosphites with functionalyzed aromatic aldehydes. Russ. J. Gen. Chem. 2005, 75, 1965-1967. [CrossRef]

32. Zhang, Y.-L.; Zhang, Y.-J.; Wang, W.-M.; Yang, K.-W. Synthesis and inhibitory activity of acetamidophosphonic acids against metallo- $\beta$-lactamases. Phosphorus Sulfur Silicon 2017, 192, 14-18. [CrossRef]

33. Safonova, T.Y.; Gulyukina, N.S.; Novakovskaya, Y.V.; Astaf'ev, E.A.; Bondarenko, G.N.; Petrii, O.A.; Tsirlina, G.A.; Beletskaya, I.P. Electrochemical hydrogenation of substituted alpha-phenylvinylphosphonic acids: General characteristics of the reaction layer and prediction of preparative electrolysis conditions. Russ. J. Electrochem. 2002, 38, 457-466. [CrossRef]

34. Barton, D.H.R.; Vonder Embse, R.A. The invention of radical reactions. Part 39. The reaction of white phosphorus with carbon-centered radicals. An improved procedure for the synthesis of phosphonic acids and further mechanistic insights. Tetrahedron 1998, 54, 12475-12496. [CrossRef]

Sample Availability: Samples of the compounds are available from the authors. 